\section{Development of a Cross-Coupled Robust Controller for a Multi-Axis Micromachining System}

\author{
Mumtazcan Karagoz \\ Department of Mechanical Engineering, \\ Bilkent University, \\ Ankara 06800, Turkey \\ e-mail: mumtazcan.karagoz@bilkent.edu.tr \\ Melih Cakmakci ${ }^{1}$ \\ Mem. ASME \\ Department of Mechanical Engineering, \\ Bilkent University, \\ Ankara 06800, Turkey \\ e-mail:melihc@bilkent.edu.tr
}

In the current era of miniaturization for complex, ubiquitous, and energy efficient systems, micromanufacturing had become one of the most popular fields for engineering development. This paper introduces a modular robust cross-coupled controller design structure applied to a three axis micromachining system that can be extended to more axis systems and configurations. In order to develop a robust controller that can withstand the disturbances due to tool-workpiece interactions, a dynamic model of the whole system is needed. Developing control-oriented models for micromachining systems can be challenging. Using the sum of sines identification input, essential nonlinearities including the effects of assembly and slider orientation are included. Verification data show that these transfer function models represent the physical system satisfactorily while avoiding an over-fit. Using the transfer functions from the identified model, a controller structure with robust axis controllers with cross-coupled control (CCC) are developed and fine-tuned with simulations. Machining experiments are also done in order to compare the performance of the proportional-integral-derivative control design, an adaptive robust controller (ARC, both from earlier work in the literature) and the new $H_{\infty}$ robust controller. According to results of experiments, the new robust controller showed the best tracking and contouring performance with improved surface quality due to reduced oscillations. [DOI: 10.1115/1.4040443]

\section{Introduction}

Starting from the early 1960 s, the most prominent trend in engineering for the search of more efficient, powerful, and cheaper devices is the miniaturization [1]. Thus, manufacturing of microdevices had become one of the most widely studied topics in the past fifty years. Even though there are new and promising methods such as laser sintering and additive manufacturing, conventional, cutting-based methods continue to hold a unique and irreplaceable position due to the desired mechanical properties of the end products. In order to successfully manufacture microscale parts, the machining system needs to be precise, due to the nature of the required features and robust, due to the nonlinearity of the cutting process with the possibility of chatter vibrations and other environmental disturbances such as electrical noise and ground vibration. Figure 1 shows the experimental machining setup used in this research. The system consists of two parts: a cast holder

${ }^{1}$ Corresponding author

Contributed by the Dynamic Systems Division of ASME for publication in the Journal of Dynamic Systems, Measurement, and Control. Manuscript received September 4, 2017; final manuscript received May 24, 2018; published online July 2, 2018. Assoc. Editor: Yang Shi. that houses the spindle (and the cutting tool) and a threedimensional positioning system that (Fig. 1(b)) moves the workpiece. In this configuration, motion in each axis is provided by a slider (Fig. 1(c)) powered by a controlled linear motor. The precise and rigid positioning of the workpiece by the sliders during the machining (milling) operation to generate a mechanical feature is considered to be a robust tracking problem.

In this application, the primary objective for the tracking control is to move the end-effector tool (the workpiece) to a predetermined point in machining space following a desired trajectory. Although almost all systems employ feedback control, considerable improvement in tracking performance can be achieved with the addition of feed-forward control to the algorithm. Several feed-forward control approaches are developed in the literature to improve tracking accuracy such as zero-phase error tracking control [2,3], feedforward friction compensation $[4,5]$, and iterative learning control [6-8].

For many applications, the tracking error is defined as the difference between the desired position and the actual position for each slider (axis). For multidimensional operations, a contour error can be calculated using geometrical relations of error vectors. This vectorial approach can be explained using the geometrical relations in Fig. 2. Here, $\mathbf{e}$ is the tracking error vector, $\hat{\boldsymbol{\varepsilon}}$ is the estimated contour error vector, $\boldsymbol{\varepsilon}$ is the contour error vector, $\mathbf{t}$ is the normalized tangential vector, $\mathbf{n}$ is the normalized normal vector, $P$ is the actual position, and $R$ is the reference (desired) position at an instant. Then, the contouring error $\varepsilon$ can be defined as the vector from the actual position to the nearest point on the line which passes through the reference position tangentially with direction $\mathbf{t}$ [9].

Generally, improving tracking accuracy of an individual motion axis (i.e., $X, Y$, or $Z$ axis) also increases the contouring accuracy of a multi-axis positioning system. However, in some cases, it is reported that decreasing the tracking error may not result in decreased contour error [10]. Hence, the control algorithm should be designed considering not only the tracking error but also the contour error. In Ref. [10], an improved cross-coupled control (CCC) structure that focuses on eliminating the contour error rather than the tracking error in individual axis is introduced. This method is proven to reduce contour error significantly. Since the introduction of the CCC, it has been modified and combined with different control techniques. Some examples can be given as the observer-based CCC [11], cross-coupled model reference adaptive control [12], CCC with disturbance observer and zero-phase error tracking control [3], CCC with friction compensation [5], and CCC with iterative learning control [7,8,13]. In Ref. [14], a proportional-integral-derivative (PID)-based robust CCC is used. The robustness of the controller is obtained by adjusting gain and phase margin of the resulting systems.

In the literature, there are studies and applications of different robust controller algorithms such as $\mu$ synthesis or adaptive robust control (ARC) to the manufacturing systems such as milling machines in macroscale. Stephans and Knospe showed that $\mu$ synthesis could be used in order to synthesize a robust controller for machining spindles [15]. Lee and Tomizuka showed that robust controllers could be used in high accuracy motion positioning systems [16]. In their work, the proposed controller have four components, a friction compensator either in the feed-forward or feedback loop, a disturbance observer in the velocity loop, a feedback controller in the position loop, and a feed-forward controller. The paper also asserts that the robustness comes from the disturbance observer portion of the proposed controller structure. Tsao and Tomizuka also showed that robust adaptive controllers could be used for noncircular machining [17]. Moradi et al. showed that by using a $H_{\infty}$ controller, chatter vibration can be significantly reduced [18]. Moreover, it is shown that, as the parameter variance increases, the effort needed to suppress the chatter vibration also increases. Also, the difference between the actual system and the modeled system greatly affects the performance of the robust controller. 


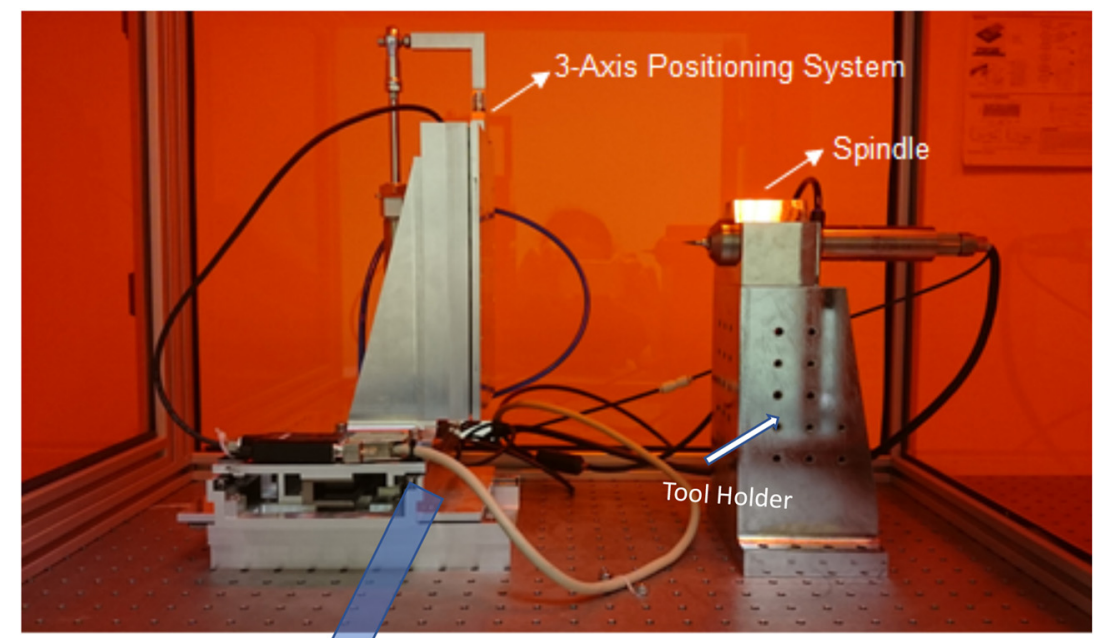

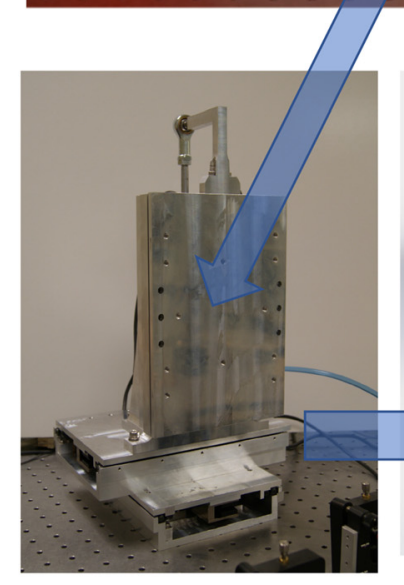

(b)

(a)

Fig. 1 Micromachining using a three axis orthogonal positioning system

\section{Contour}

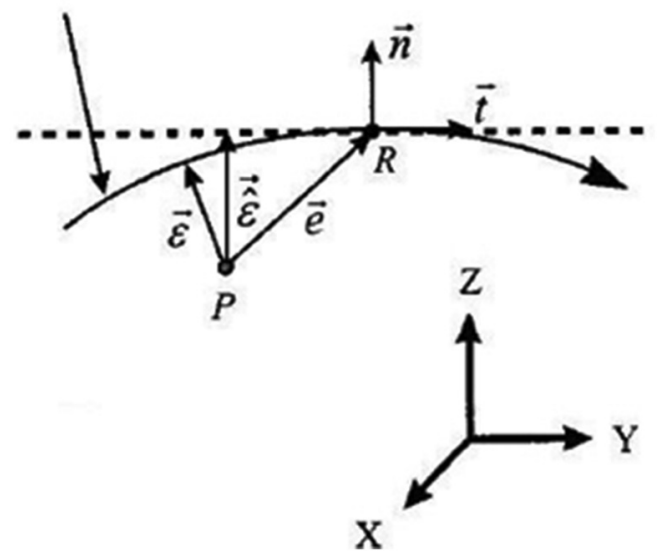

Fig. 2 Contour error versus tracking error

This paper presents our work on designing a $H_{\infty}$ robust $\mathrm{CC}$ control algorithm that is suitable for micromachining applications. There are two main contributions of this work: first contribution is a mathematical model for an orthogonal three axis positioning system that includes the effect of assembly and device nonlinearities rather than using individual slider models for each axis based on ideal laws. This is a challenging task considering the short stroke (only $110 \mathrm{~mm}$ ) of the axis sliders and typical identification inputs cannot work efficiently. Second contribution is a $H_{\infty}$ based robust controller design structure that utilizes cross-coupled control to include the effects of other axis dynamics. To the best of our knowledge, this is the first comparative study in the literature that combines an optimal robust controller with CCC for a micromachining system with experimental results. In Sec. 2, modeling and identification of the positioning system from its axis modules will be discussed. In Sec. 3, a method to design the control algorithm for tracking and robustness will be discussed with simulations. The machining experiments performed to validate the functionality of the designed system will be presented in Sec. 4. In Sec. 5, our current results and future work will be discussed.

\section{Modeling and Identification}

In order to synthesize a position tracking controller, a mathematical model for the workpiece positioning system should be developed. The first approach can be a physics-based system modeling approach using lumped parameters. However, due to the complex nature of the electromechanical positioning system this cannot be enough for precision and robustness. In the following section, the development of the baseline model and then the identification of the system are explained.

2.1 Physics Based Modeling. Figure 1 shows the experimental machining setup used in this research. The axis sliders are operated by linear motors, and the displacement is measured using optical encoders as shown in Fig. 1(c). One approach to model this orthogonal $X Y Z$ positioning configuration is to assume the system as a perfect assembly of three separate but identical single axis subsystems.

A physics-based lumped parameter model developed for nanopositioning (nonmachining) applications were explained in 
Ref. [8]. Using this approach, a second-order transfer function can be obtained as shown in the following equation where $X(s)$ is the slider displacement, and $E(s)$ is the voltage command to the system

$$
\frac{X(s)}{E(s)}=\frac{k_{M}}{L m_{i} s^{3}+\left(R m_{i}+b_{i} L\right) s^{2}+\left(R b_{i}+k_{M}^{2}\right) s}
$$

In Eq. (1), $L, R, k_{M}$, are the linear motor inductance, resistance, and motor constant, respectively. $m_{i}$ and $b i$ represent the inertia of the slider $i$ and the viscous friction experienced by the linear bearings. Even though the sliders are assembled from identical components, due to the configuration of the positioning system, the value of the inertia and friction constant may change. For example in our system, the $X$-axis slider carries $Y$-axis and $Z$-axis system as well requiring different mass and viscous friction parameters. This model could be used to calculate a theoretical robust controller using the experimental values from Ref. [8]. However, during the actual implementation, this model is known to have various problems under the influence of moderate disturbance. First of all, the model presented in Eq. (1) uses a viscous friction model, whereas the micromachining system shows stick-slip like behavior [6]. Furthermore, the single axis slider model with the transfer function given in Eq. (1) assumes $X, Y$, and $Z$ axes sliders are identical and have exactly the same contribution to the position of the end effector (in different directions) given the same input. This, however, is observed to be deceiving based on the actual experiments and sensor data due to the slider manufacturing and assembly conditions. Finally, unmodeled dynamics, especially for the linear motor electronics, could have significant contributions on the system response. Therefore, an identification procedure where the tests are performed on the complete assembly rather than the axis sliders is preferred. Based on the physical model given in Eq. (1), at least second-order transfer functions will be assumed for system dynamics.

2.2 System Identification. System identification is a procedure that consists of measuring input and output data of any given system and calculating a mathematical model that emulates the response of the system based on this data. The identification input must be carefully selected since it needs to be complex enough to extract meaningful information (i.e., to achieve persistent excitation) while complying with the limitations on the data acquisition system. Furthermore, the identified model needs to fit closely to the actual system while avoiding over-fitting. In this work, the measurements are taken by using linear optical encoders utilizing an adaptive correction and look-up table-based interpolation method discussed in Ref. [19]. Stable resolutions as low as $60 \mathrm{~nm}$ using linear optical encoders and interpolation were obtained for slider speeds up to $45 \mathrm{~mm} / \mathrm{s}$.

During the experiments, all three directions of the system $(X, Y$, and $Z$ ) are excited by the identification input separately, gathering $X, Y$, and $Z$ axis displacement data. The cross-dynamics effects between the axes are observed to be small and are currently disregarded. For each data set ( $X, Y$, and $Z$ axes), the identification tests are repeated five times with different starting points on the slider. For transfer function identification and verification, the averaged data of these repetitions are used.

There are several options for inputs that can be used in system identification experiments such as sweep, random, or sum of harmonic signals [20]. Each of these inputs has their own advantages. When the application is considered, using a sweep signal is not feasible since the operation range of the sliders is limited. The random signal input was not preferred since each of the axes is identified individually making repeatability an important factor. Therefore, the sum of sines input, which excites the system over the displacement range, is selected. As a result, the final identification signal is a highly complicated periodical signal in time domain. For verification of the identified model, an input signal with the same frequency content but with different magnitudes is used. In Figs. 3(a) and 3(b), the identification and verification signals used for each axis are given, respectively. The transfer functions calculated from the input signal data are then compared to the verification signal data.

The magnitude of the identification signal was varied for each axis to cover all available range while avoiding the limits of the slider motion. $X, Y$, and $Z$ axes are excited individually while logging the position data of all axes. Then, using these data, transfer functions $G_{x x}=X(s) / U_{x}(s), G_{y y}=Y(s) / U_{y}(s), G_{z z}=Z(s) / U_{z}(s)$ are identified.

The level of fit of identified transfer functions and the measured data set is calculated with normalized root-mean-square (RMS) method. Using the system output of $X$ axis and prepared identification input, system model is calculated using MATLAB's System Identification Toolbox. In frequency domain, simulated system output fits to the measured system output with \%86.3. Using the validation data set, simulated system output fits to the measured system output \%84.2. The identified transfer functions are validated in time domain. As shown in the Fig. 4(a), simulated system output and measured system output are close to each other with a $\% 71.9$ fit. In comparison, simulated response fit to the measured verification data fairly well as shown in Fig. 4(b) with a fit of $\% 68.2$.

After completing the experiments and analysis, the identified transfer function for $X$-axis, $G_{x x}$, is given below:

$$
G_{x x}=\frac{56930 s+1389}{s^{2}+0.2268 s+0.002058}
$$

As the second and third steps of the identification process, the input signals are applied to the slider providing motion on the $Y$ axis and $Z$-axis separately, and the motion of all axis sliders was recorded. Then, the best fit for both $G_{y y}$ and $G_{z z}$ were found using the System Identification Toolbox as shown in Eqs. (3) and (4), respectively. All the identified transfer functions fit approximately $\% 70$ in the frequency domain, and the results of the verification tests show around \%68 fit for both of the $Y$ and $Z$ axes cases. Therefore, it was concluded that acceptable fits are obtained while avoiding over fit without having to use high-order polynomials. Identified transfer functions are verified in the time domain as presented in Fig. 4(b)

$$
\begin{gathered}
G_{y y}=\frac{548000 s+13670}{s^{3}+1.644 s^{2}+8.198 s+0.06571} \\
G_{z z}=\frac{355600 s+5468000}{s^{3}+607.5 s^{2}+344 s+2643}
\end{gathered}
$$

\section{Controller Design and Simulations}

In this section, primary feedback controllers for each axis are designed using the mathematical models obtained in the previous section. These controllers are then combined with the crosscoupled controller and performance simulations were performed. For comparison, the design of a PID-CC [8] and ARC [16] from Sec. 2 literature is also discussed and simulated.

3.1 $\boldsymbol{H}_{\infty}$ Controller Design. The generic $H_{\infty}$ control problem structure is given in many sources in the literature such as Ref. [21]. In this standard formulation $w, z, u$, and $y$ are the external input, output, control signal, and measured variables, respectively. A transfer function matrix $F_{l, i}\left(G_{i i}, C_{i i}\right)=T_{z w}^{i}$ can be defined from external inputs $w$ to output $z$ where $G_{i i}$ is the axis model developed for $X, Y$, and $Z$ in Sec. 2 and $C_{i i}$ is the corresponding robust controller. Then, $z$ can be derived as $z=F_{l, i}\left(G_{i i}, C_{i i}\right) w=T_{z w}^{i} w$. The $H_{\infty}$ optimization problem is to find a stabilizing controller $K$ 

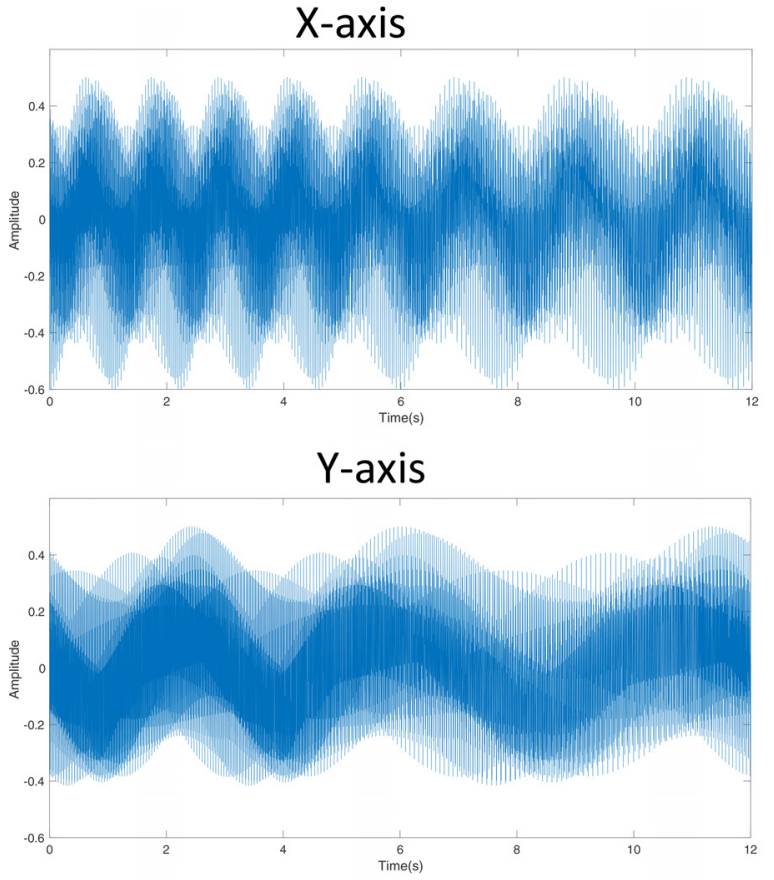

Z-axis

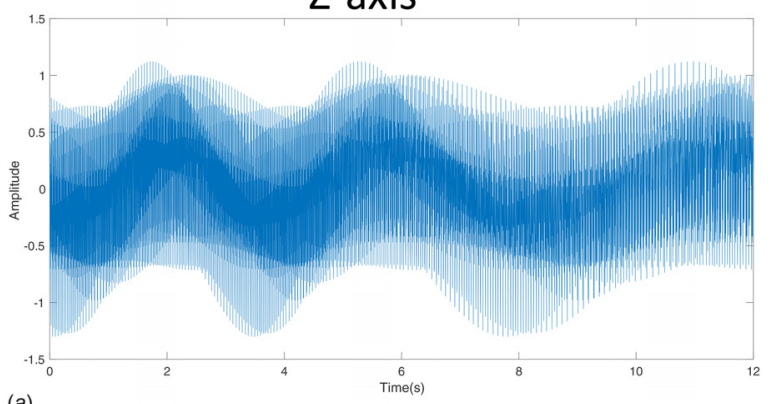

X-axis
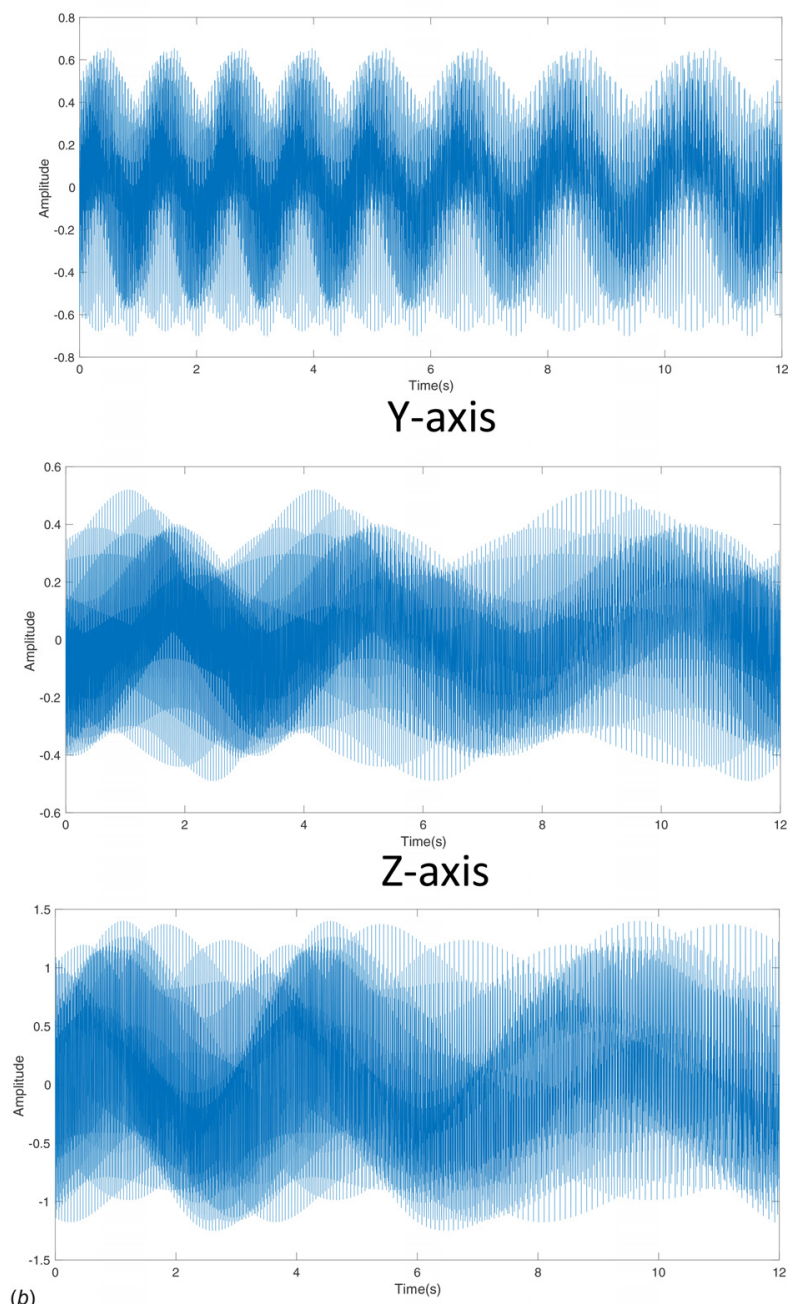

Fig. 3 (a) Identification and (b) verification Inputs

that will minimize the infinity norm of the $T_{z w}^{i}$. This can be expressed mathematically as shown in the below equation:

$$
\min _{C_{i i} \in H_{\infty}}\left\|T_{z w}^{i}\right\|_{\infty}=\min _{C_{i i} \in H_{\infty}}\left\{\sup _{\operatorname{Re}(s)>0} \bar{\sigma}\left[T_{z w}^{i}(s)\right]\right\}
$$

The optimal $T_{z w}^{i}$, can be tuned to have distinct behaviors on different frequency bands for each axis. Commonly used performance measures are the sensitivity, $S$, and complementary sensitivity, $T$, functions for the closed-loop system $(S+T=I)$. The weight functions $W_{s}^{i}$ and $W_{t}^{i}$ can also be implemented on the system to obtain the inequality constraint in the following equations:

$$
\left\|T_{z w}^{i}\right\|_{\infty}=\left\|\begin{array}{l}
W_{s}^{i} S \\
W_{t}^{i} T
\end{array}\right\|<\gamma
$$

$W_{s}^{i}$ and $W_{t}^{i}$ are constructed to achieve desired goals within desired frequency bands. For instance, for most cases, $S$ is minimized at low frequencies to achieve better tracking and disturbance attenuation, where $T$ is the minimized at high frequencies to achieve robust stability in the presence of sensor noise, variations in the system parameters, and unmodeled high-order dynamics. In order to synthesize a $H_{\infty}$ controller for the system, first of all the weight functions need to be selected. Even though guidelines for the weight function design exist, fine-tuning using the simulations is generally necessary. Using a weight function formulation suggested in Ref. [21], we obtain the formulations given in the below equations to synthesize the axis controllers

$$
\begin{aligned}
& W_{s}^{i}=\frac{s / M+\omega_{0}}{s+\omega_{0} A} \\
& W_{t}^{i}=\frac{\omega_{0} / M+s}{A s+\omega_{0}}
\end{aligned}
$$

where $\omega_{0}$ is the desired closed-loop bandwidth, $M$ is the noise sensitivity peak, and $A$ is the target steady-state offset. These weight functions tuned manually using simulations based on the guidelines given in Ref. [21] as presented in Table 1.

Using the weight functions, robust $H_{\infty}$ controllers are designed using MATLAB Robust Control Toolbox for each axis. The resulting $H_{\infty}$ axis controllers for the $X, Y$, and $Z$ axes are shown in the following equations:

$$
\begin{gathered}
C_{x x}=\frac{525630(s+16000)(s+0.22)(s+0.01)}{(s+56930000)(s+52660)(s+1.6)(s+0.03)} \\
C_{y y}=\frac{2881(s+180000)(s+0.01)\left(s^{2}+1.64 s+8.12\right)}{(s+177300)(s+30900)(s+420)(s+0.15)(s+0.03)} \\
C_{z z}=\frac{1121055000(s+12000)(s+607)\left(s^{2}+0.56 s+4.354\right)}{(s+38920)(s+15.4)(s+1.2)\left(s^{2}+29570 s+358500000\right)}
\end{gathered}
$$



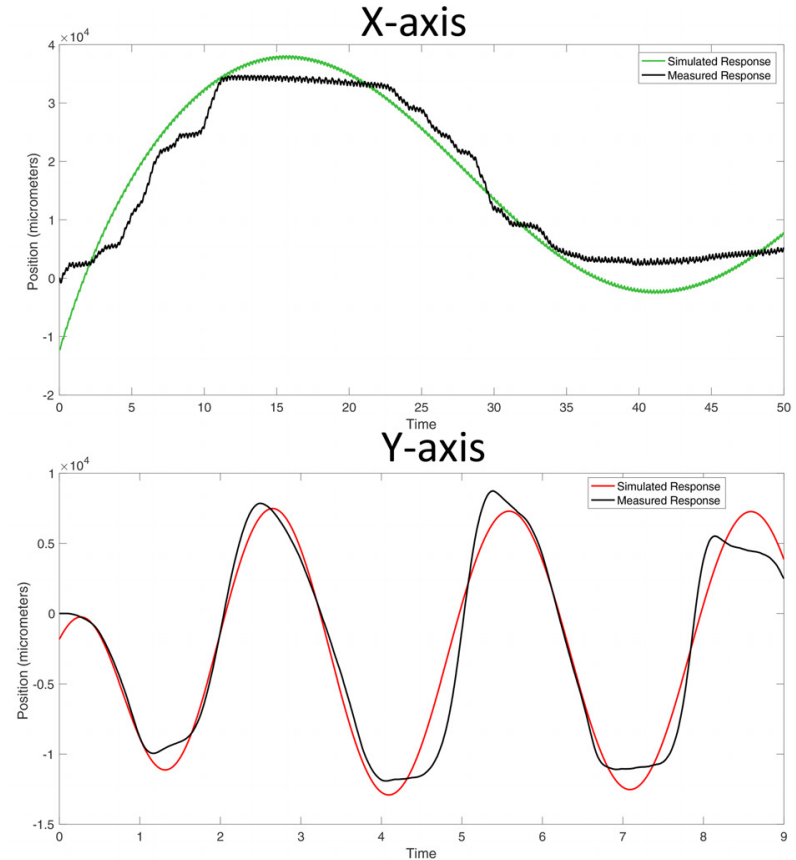

Z-axis

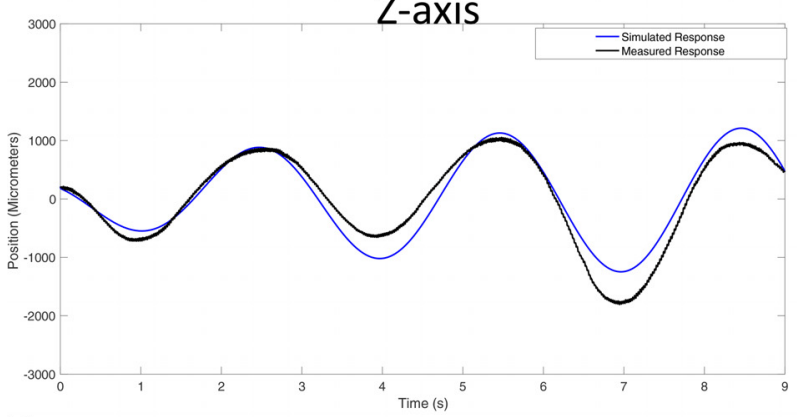

(a)

Fig. 4 Response in time domain: (a) identification and (b) verification
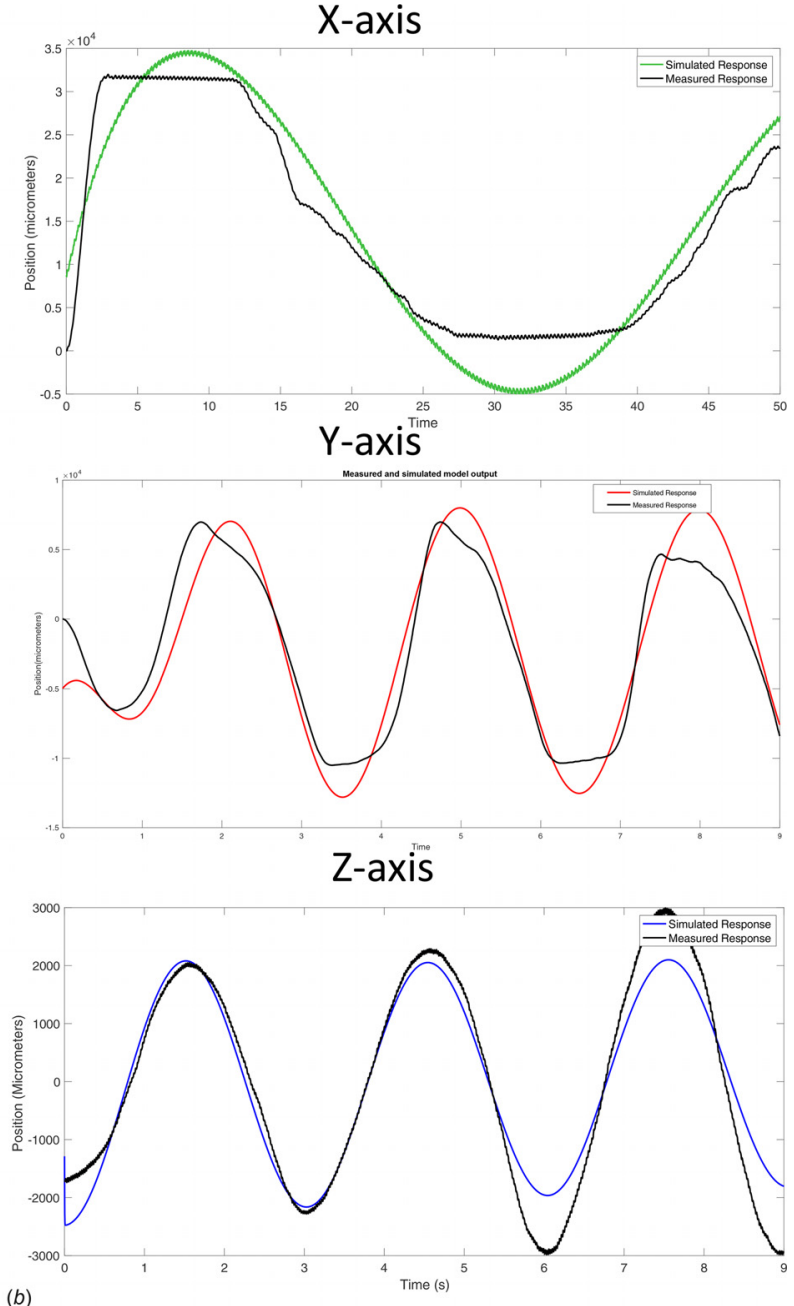

(b)
Table 1 Weight function parameters for each axis

\begin{tabular}{lcccc}
\hline \hline Axis & $A$ & $M$ & $\omega_{0 t}$ & $\omega_{0 s}$ \\
\hline$X$ & 0.001 & 2 & 160 & 160 \\
$Y$ & 0.001 & 2 & 150 & 180 \\
$Z$ & 0.01 & 2 & 120 & 120 \\
\hline \hline
\end{tabular}

Table 2 PID parameters (best set)

\begin{tabular}{lllcl}
\hline \hline PID parameters & $P$ & $I$ & $D$ & $N$ \\
\hline$X$ & 1 & 0.01 & 0.0005 & 100 \\
$Y$ & 1.5 & 0.01 & 0.0005 & 100 \\
$Z$ & 1 & 0.01 & 0.0005 & 100 \\
$\mathrm{CC}$ & 0.4 & 0.005 & 0.0005 & 100 \\
\hline \hline
\end{tabular}

3.2 Proportional Integral Derivative-Cross Coupled Controller. In order to compare the performance of the newly identified model and the $H_{\infty}$ robust controller, various alternatives from the literature is used. As a first step, the cross-coupled PID controller from Ref. [8] is used. This controller is extensively used in this system per previous research, and the optimized parameters are given in Table 2 where $P, I, D$, and $N$ are the proportional, integral, derivative, and filter constants, respectively.

3.3 Cross Coupled Adaptive Robust Controller. In Ref. [16], a widely cited ARC for high accuracy motion positioning systems is presented. The ARC has four components, a friction compensator either in the feed-forward or feedback loop, a disturbance observer in the velocity loop, a feedback controller in the position loop, and a feed-forward controller. As a comparison for our controller, an ARC controller was developed for each axis. For the loop controller PID controller from Sec. 3.2 was used. Then, for the disturbance observer, the inverse of the identified axis dynamics from the modeling sections were used with a filter $Q(s)$ as described in the paper with the prescribed values for $G_{d}(s)$ as the friction based feed-forward controller. The cross-coupled controller structure and parameters from Sec. 3.2 is used for comparison.

3.4 Simulations. In Fig. 5, the block diagram of the control structure used in simulations and in the experimental setup is shown. The axis controllers are used as either PID from Ref. [8], ARC from Ref. [16], or the robust $H_{\infty}$ controller developed in this paper. These controllers take desired $x_{d}, y_{d}$, and $z_{d}$ position reference and determine the linear motor actuation command based on the error. The ARC controller structure also uses the reference input for its feed-forward controller. The milling tool operates the cutting tool at desired angular speed. For this study, a constant speed is set during cutting experiments.

In order to carry out the simulations, a system model is created in MATLAB/SIMULINK using the transfer functions identified previously. The input tracking signals $x_{d}, y_{d}$, and $z_{d}$ are created using $S$ curves [19] with a MATLAB script offline for $X, Y$, and $Z$ axes, respectively. For this study, a maximum acceleration of 


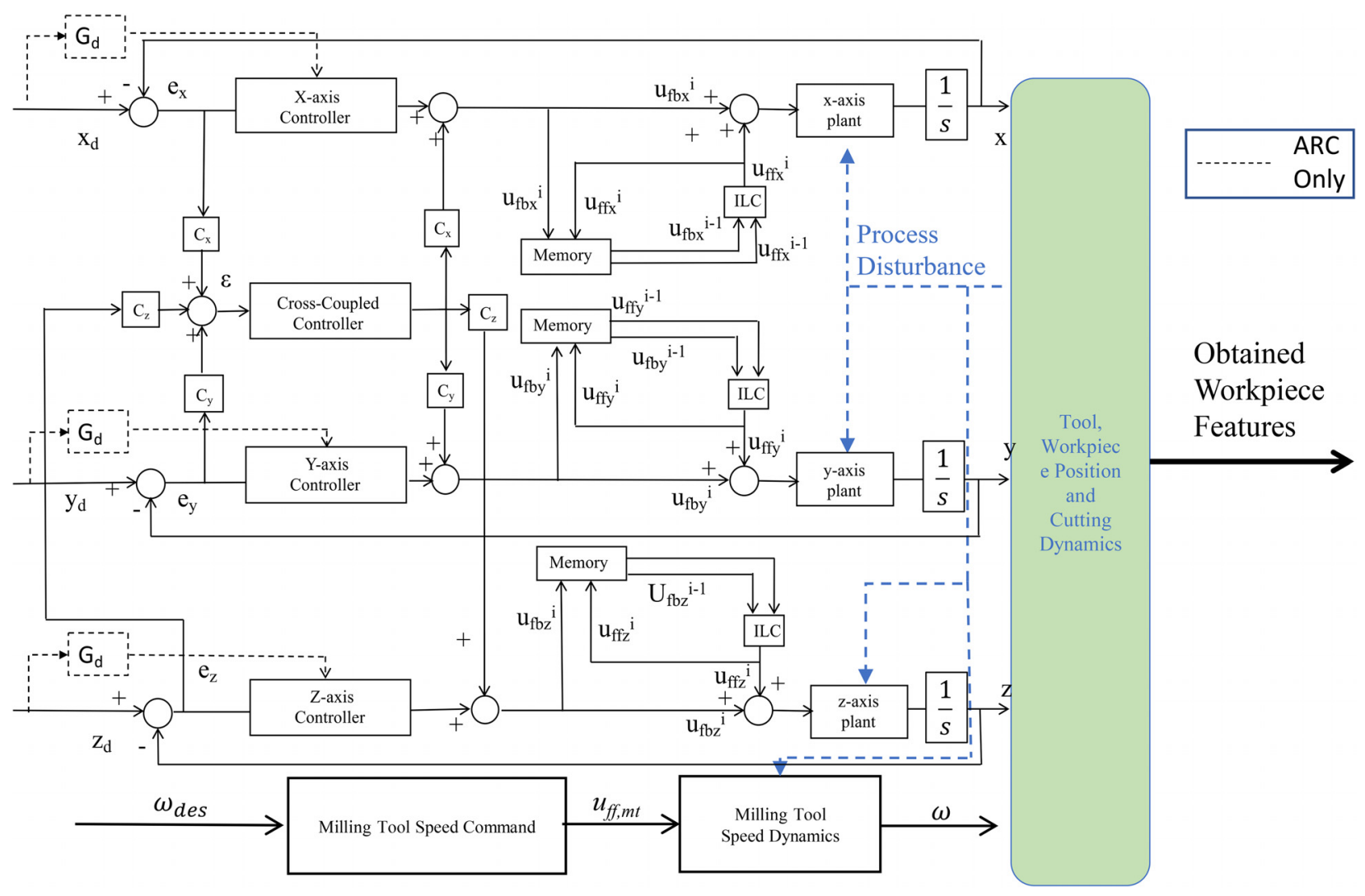

Fig. 5 Block diagram of the controller structure used with the micromachining system

$1.5 \mathrm{~mm} / \mathrm{s}^{2}$ and a maximum velocity up to $30 \mathrm{~mm} / \mathrm{s}$ is applied when the curves are designed. Generated input signal is fed into the simulation setup as the reference value, and the output data are recorded for analysis. In order to measure the performance of the controllers both axis position errors, $e$, and contour error, $\varepsilon$, are used. The controller structure presented in Fig. 5 includes an axis based iterative learning controller. As mentioned in Ref. [8], the tracking performance of the system generally gets better with more iterations. For the initial simulation, the desired shape is an inclined circle with a diameter of $5 \mathrm{~mm}$ and an incline angle of $45 \mathrm{deg}$. For the simulations and experiments presented in this paper, the first run results are given as the expected worst case scenario without using the iterative controllers.

To test robustness, the simulations are run using a sinusoidal disturbance input in each axis. The amplitude of the disturbance is selected as $100 \mu \mathrm{m}$ and the frequency is $75 \mathrm{rad} / \mathrm{s}$. This position disturbance is injected to system after the plant. The results given in Table 3 show the error obtained for each axis and the contour error, $\varepsilon$.

Based on the error values given in Table 3 , all error components are improved by using a robust controller. The significant decrease of the RMS errors show that robust controllers (ARC and $H_{\infty}$ ) disturbance rejection property is far better than the PID controller. For the simulations, it can be seen that the adaptive robust controller from Ref. [16] performed better with the new robust controller not far behind. Also, none of the axis RMS error

Table 3 RMS errors for different controllers in $(\mu \mathrm{m})$

\begin{tabular}{lccc}
\hline \hline & PID-CC [8] & ARC-CC [16] & $H_{\infty}$-CC \\
\hline$e_{x}$ & 146.7 & 35.1 & 42.1 \\
$e_{y}$ & 138.8 & 42.3 & 74.7 \\
$e_{z}$ & 146.7 & 51.7 & 52.1 \\
$\epsilon$ & 108.3 & 58.4 & 69.0 \\
\hline \hline
\end{tabular}

values for robust controllers is greater than the amplitude of disturbance signal. According to the simulations results, one can conclude that robust controllers are far more suited to a machining system where many unavoidable disturbance signals are present.

\section{Experiments With the Micromachining System}

In order to evaluate the real-life performance of the machining system, several experiments conducted using both the PID-CC, ARC-CC, and $H_{\infty}$-CC controllers. Our experimental micromachining system consists of a three-axis orthogonal positioning system and micromilling spindle as shown in the Fig. 1(a). As was done with the simulations, the three axis-positioning system was controlled using different controllers for each case, and the position data were collected. Milling spindle currently has its own speed controller, which can be set between 1 and $60 \mathrm{krpm}(25$ krpm for the tests).

Implementation of the controllers is done using the NI LABVIEW software. For the experiments presented in this section, the first run results are given as the expected worst case scenario without using the iterative learning controllers. For the experiments, several input signals (cutting paths) are generated. These input signals are generated using S-curves in order to limit the jerk to have a smooth path for the positioning system. The usage of S-curve also minimizes the effects of cross-coupling due to the inertia of the sliders.

The primary cutting test is the square cutting experiment as described in Fig. 6. It should be noted that since square shape has sharp turns at corners, the disturbance caused by the cross axis effects has a larger effect compared to the circular cutting test which consists of only sinusoidal motion for each $X$ and $Y$ axis. The length of a side of the square to be cut is $5 \mathrm{~mm}$. A depth of $200 \mu \mathrm{m}$ is designed for square cutting test, but because of the manual homing of the system, final depth of the manufacturing is error prone.

To compare the performance of each controller, the position error data are calculated and tabulated in Table 4. Our 


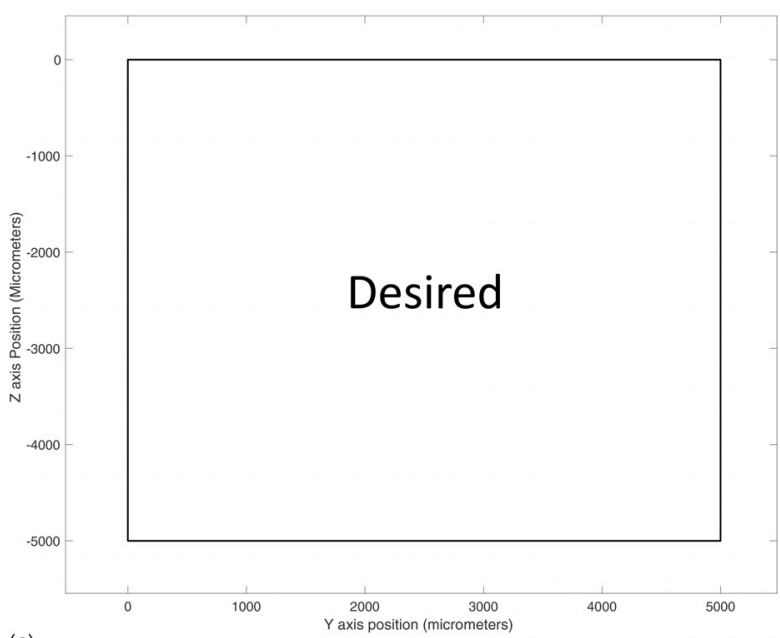

(a)

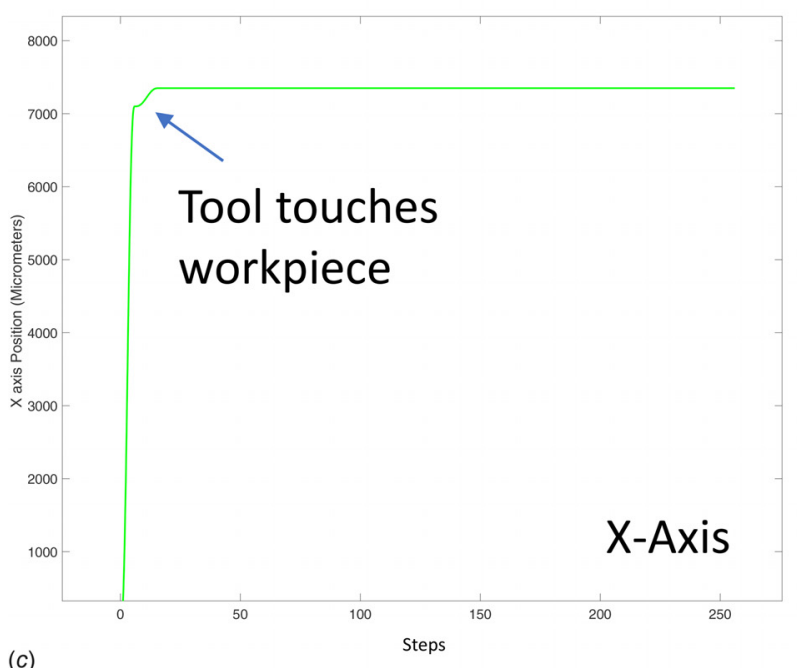

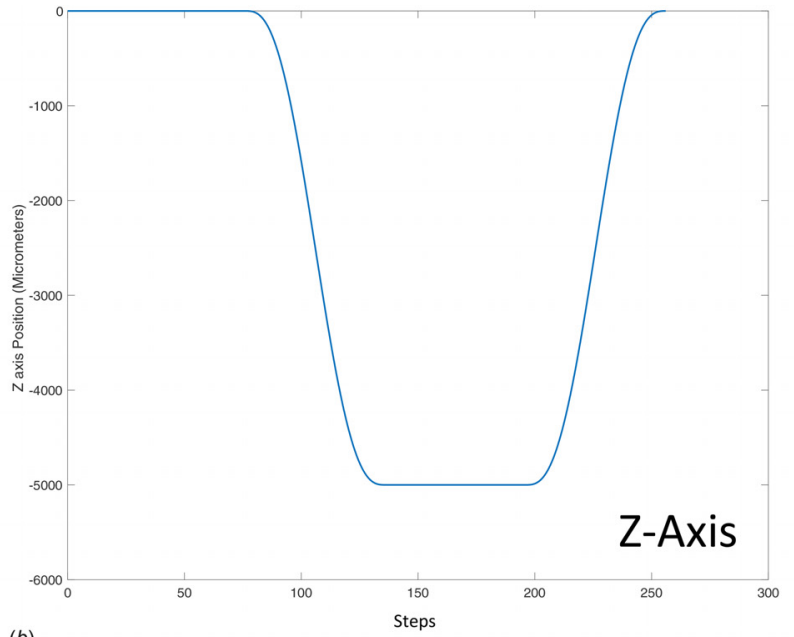

(b)

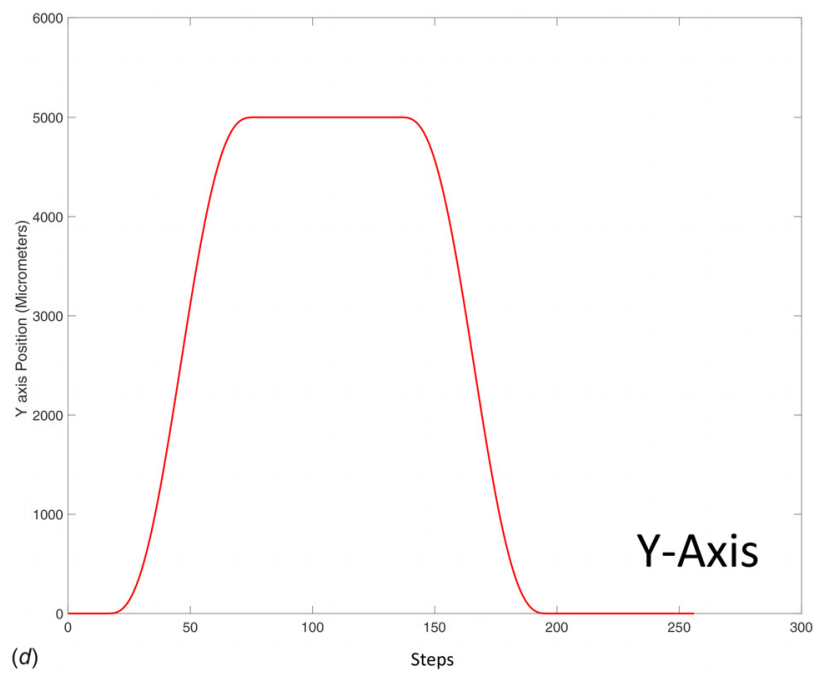

(d)

Fig. 6 (a) Desired square profile, (b) Z-axis, (c) X-axis, and (d) $Y$-axis motion profiles

experimental axis and contour error data show that the PID-CC controller performed the worst for both axis and contour errors. The newly developed $H_{\infty}$-based robust controller performed the best in all aspects that also showed similar error magnitude as compared to simulation results. The ARC-CC controller performed much better than the PID-CC controller and its performance is comparable with the $H_{\infty}$ controller. Although its good performance is well documented in many sources, we believe the disturbance observer component (the third-order plant inverse and compensation with filter $Q(s)$ ) may have played a role the lower performance in this case.

Because of the oscillations, there are several unsuccessful cutting attempts during our experiments. The image in Fig. 7(a) shows such a drastic case for our circular cut tests where the instability in the controller, albeit recovered later, caused problems. The artifacts in the surface quality can be easily seen in the optical microscopy image.

Table 4 RMS errors for different controllers in $(\mu \mathrm{m})$ square cutting experiments

\begin{tabular}{lccc}
\hline \hline & PID-CC [8] & ARC-CC [16] & $H_{\infty}$-CC \\
\hline$e_{x}$ & 257.3 & 97.1 & 83.0 \\
$e_{y}$ & 242.1 & 109.2 & 87.7 \\
$e_{z}$ & 211.6 & 110.7 & 67.1 \\
$\epsilon$ & 190.1 & 102.6 & 89.3 \\
\hline \hline
\end{tabular}

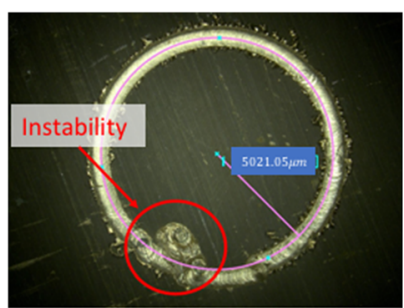

(a)

PID-CC Controller
Fig. 7 Optical microscope images cut samples: (a) PID-CC controller and $(b) H_{\infty}-C C$ controller

In our best cutting performance, according to the optical microscope image of the square cut shown in Fig. 7(b), the side lengths of the square are $4997 \mu \mathrm{m}$ with the newly developed controller that performed best among all controllers in the average. Measured depth is $182 \mu \mathrm{m}$. Even with a smooth S-curve, the square cutting test has sharp turns at the edges. The sharp turns, the fact that the controllers were built directly using the identified plant functions which include the effect of cross-coupling could be the reason of this large decrease in side length error in robust controller.

\section{Conclusions}

In this paper, a cross-coupled $H_{\infty}$ robust controller structure suitable for micromachining applications was presented for 
modular orthogonal systems. $H_{\infty}$ robust controllers for $X, Y$, and $Z$ axis's are synthesized using both the identified transfer functions and tuned weighting functions. An ARC based on [16] was also developed and used for comparison. Simulation results showed both robust controller structures give significant improvements (up to \%70), under the effect of disturbance, as compared to a PID controller with cross-coupling compensation. The controllers are then implemented on the micromachining system to conduct cutting experiments. A significant performance improvement is obtained with the newly developed controller with good surface properties. There is a significant decrease in the error in square (complex due to sharp turn) cutting test with $H_{\infty}$ robust controller which performed the best in accumulated error performance metrics as well. The adaptive robust controller performed significantly better than the PID-CC controller; however, we believe the nonlinear and short range nature of the sliders affected its final performance. Furthermore, there are significantly less erroneous features on the manufactured parts when using both robust controllers. The future work on this topic is to include the milling tool speed into the controller loop and to improve the cross-coupled controller algorithm to achieve better machining performance.

\section{Acknowledgment}

The equipment used in this research is sponsored by Scientific and Technical Research Council of Turkey (TUBITAK) through Project No. 113M172. The authors would like to thank Dr. Yigit Karpat, Dr. Sakir Baytaroglu, and Dr. Samad Oliaei for their invaluable support.

\section{References}

[1] Feynman, R., 1960, “There's Plenty of Room at the Bottom: An Invitation to Enter a New Physics," Eng. Sci., 23(5), pp. 22-36.

[2] Tomizuka, M., 1987, "Zero Phase Error Tracking Algorithm for Digital Control,” ASME J. Dyn. Syst. Meas. Control, 109(1), pp. 65-68.

[3] Qing, L., Tai-Yong, W., Ying-Chuan, D., Yong-Xiang, J., and Bo, L., 2010, "Applications of Position Controller for CNC Machines Based on State Observer and Cross-Coupled Controller," IEEE International Conference on Computer Mechatronics, Control and Electronic Engineering (CMCE), Changchun, China, Aug. 24-26, pp. 593-596.

[4] Tomizuka, M., 2007, "Friction Compensator for Feed Drive Systems Consisting of Ball Screw and Linear Ball Guide," 35th International MATADOR Conference, Taipei, Taiwan, July, pp. 311-314.
[5] Wang, L., Lin, S., and Zeng, H., 2011, "Precision Contour Control of XY Table Based on LuGre Model Friction Compensation," IEEE Second International Conference on Intelligent Control and Information Processing (ICICIP), Harbin, China, July 25-28, pp. 1124-1128.

[6] Tan, K. K., Dou, H., Chen, Y., and Lee, T. H., 2001, "High Precision Linear Motor Control Via Zero-Phase Filtering," IEEE Trans. Control Syst. Technol., 9(2), pp. 244-253.

7] Barton, K. L., and Alleyne, A. G., 2008, "A Cross-Coupled Iterative Learning Control Design for Precision Motion Control," IEEE Trans. Control Syst. Technol., 16(6), pp. 1218-1231.

[8] Gecer-Ulu, N., Ulu, E., and Çakmakc i, M., 2016, "Design and Analysis of a Modular Learning Based Cross-Coupled Control Algorithm for Multi-Axis Precision Positioning Systems," Int. J. Control Autom. Syst., 14(1), pp. 272-281.

[9] Yeh, S.-S., and Hsu, P.-L., 2002, "Estimation of the Contouring Error Vector for the Cross-Coupled Control Design," IEEE/ASME Trans. Mechatronics, 7(1), pp. 44-51.

[10] Koren, Y., and Lo, C. C., 1991, "Variable-Gain Cross-Coupling Controller for Contouring," CIRP Ann. Manuf. Technol., 40(1), pp. 371-374.

[11] Naumovic, M., and Stojic, M., 1997, "Design of the Observer Based CrossCoupled Positioning Servodrives," IEEE International Symposium on Industrial Electronics (ISIE '97), Guimaraes, Portugal, July 7-11, pp. 643-648.

[12] Chuang, H. Y., and Liu, C. H., 1990, "A Model Referenced Adaptive Control Strategy for Improving Contour Accuracy of Multi-Axis Machine Tools," Conference Record of the 1990 IEEE Industry Applications Society Annual Meeting, Seattle, WA, Oct. 7-12, pp. 1539-1544.

[13] Li, H. S., Zhou, X., and Chen, Y., 2005, "Iterative Learning Control for Cross-Coupled Contour Motion Systems," IEEE International Conference on Mechatronics and Automation, Niagara Falls, NY, July 29-Aug. 1, pp. 1468-1472.

[14] Yeh, S.-S., and Hsu, P.-L., 1999, "Theory and Applications of the Robus Cross-Coupled Control Design," ASME J. Dyn. Syst. Meas. Control, 121(3), p. 524.

[15] Stephens, L. S., and Knospe, C. R., 1996, “ $\mu$-Synthesis Based, Robust Control Design for AMB Machinig Spindles," Fifth International Symposium on Magnetic Bearings, Kanazawa, Japan, Aug. 28-30, pp. 153-158.

[16] Lee, H. S., and Tomizuka, M., 1996, "Robust Motion Controller Design for High-Accuracy Positioning Systems," IEEE Trans. Ind. Electron., 43(1), pp. 48-55.

[17] Tsao, T.-C., and Tomizuka, M., 1994, "Robust Adaptive and Repetitive Digital Tracking Control and Application to a Hydraulic Servo for Noncircular Machining," ASME J. Dyn. Syst. Meas. Control, 116(1), pp. 24-32.

[18] Moradi, H., Movahhedy, M. R., and Vossoughi, G. R., 2009, "Robust Control Strategy for Suppression of Regenerative Chatter in Turning," J. Manuf. Process., 11(2), pp. 55-65.

[19] Ulu, E., Gecer Ulu, N., and Cakmakci, M., 2014, "Development and Validation of an Adaptive Method to Generate High-Resolution Quadrature Encoder Signals,” ASME J. Dyn. Syst. Meas. Control, 136(3), p. 034503.

[20] Kiong, T. K., Heng, L. T., and Sunan, H., 2008, Precision Motion Control, Vol. 49 of Advances in Industrial Control, Springer, London.

[21] Beaven, R., Wright, M., and Seaward, D., 1996, "Weighting Function Selection in the $h_{\infty}$ Design Process," Control Eng. Pract., 4(5), pp. 625-633. 